\title{
Alexander Blankenagel Alles zugleich kann man nicht ändern: Neues Denken auf alten Selbstverständlichkeiten in der UdSSR*-
}

\author{
1. Methodische Vorbemerkungen \\ 1. Der theoretische Ansatz
}

Die augenblicklichen Änderungen im Gesellschafts- und Rechussystem der UdSSR werden wesclicherseits allgemein mit Interesse, Anteilnahme und wohl meist guten Wünschen verfolgt; es srellen sich in diesem Zusammenhang natürlich auch alle möglichen Fragen. Eine dieser Fragen betriffr die unsichtbaren und unbewußeen Bremsen dieses gesellschaftichen Innovarionsprozesses; ihnen möchre ich meine Aufmerksamkeit widmen.

Änderungen im Rechissystem, ganz gleich ob im materiellen Reche oder in den juristischen Institutionen, betreffen immer die Änderungen gesellschaftlicher Strukturen. Man weiß seit langem, daß, will man eine Gesellschaft ändern, man natürlich Strukturen ändern muß: Eine Ändenung der Strukturen allein reicht aber nicht aus. Ebenso wichrig sind die diese Strukturen und Inscitutionen umlagernden Einscellungen, die in der Politikwissenschaft als "politische Kultur * bezeichnet werden.' Diese umlagernden Einstellungen bestimmen den konkresen Modus des Funktionierens von Institutionen und Strukturen, den Grad ihrer Akzeptanz oder Umgehung, ihr Leben und auch ihr Sterben ebenso wie das Entstehen nspontaser * gesellschaftlicher Umgehungsinstitutionen. Dabei geht es freilich in der Politikwissenschaft, anders als in der Alltagssprache, niche um eine qualitative Bewernung dieser Einselliungen, sondern nur um ihre empirische Ermittlung: Niche die hohen und die niedrigen, die verwerflichen und die demokratisch begrüßenswerten Einstellungen sind interessant, sondem schliche die konkreten Inhalte der Einstellungen als soicher. Im Laufe der politikwissenschaftlichen Diskussion ist das Konzept in unterschiedlicher Richrung weiterentrvickelt worden: politische Symbolik, politische Kommunikation und Sozialisation sind einbezogen worden. ${ }^{2}$ Man hat des weiteren auch versuch,, über

\footnotetext{
"Dic folgenden Ausfïhrungen beruhen z. T. aul einer empirsschen Untersuchung, dic ich im Herbst 1987 in Moskau durchgeführe habe. Dank geht an die DFG für die Gewährung des Farschungssupendiums und an die sowjenschen Gesprächspanner, die sıch bereırwillig stundenlang von mur interviewen heßen. Mehr als Dank gehe freilich an V. M. Saviekij, ohne dessen Verbnndungen (und Überredungskünsre) die von mır geplanten Interviews sıcherlich mangels Intcrvicwpartener geschcıtcr wären. Dic Venófiedelichung stellt den mut Fußnoten versehenen Text meiner Wurzbueger Antrituvarlesung aus dem Sornmerscmester 1988 dari dic Vortrassiorm aurde größrenteils bcibehalien.

I Zu dem Ásale siehe etwa G. A. Almond, Comparauve Policical Systems, in: JPol 18 (1956), 3916 . L.W. Pye/S. Verba, Polincal Culeure and Poliucal Developmene, 1965: G. A. Almond/S. Verbu, The Cinc Culture, 1963; dies., The Civic Culture Revisited, 1980; zur deutschen Rezepeson s. var allem die verhälenismäßig frulie Arbeıt von D. Berg-Schlosser, Die polinische Kulnur, 1972.

2 S. vor allem L. Diemer, Political Culture and Polincal Symbolism: Toward a Theoreucal Synebess, in: World Poliues XXIX (r977), s2 II.
} 
dic Momentaufnahme der politischen Kultur hirzaus mit dem Konzept "gesellschaftlicher Codes" die Entwicklungsformeln konkreter Gesellschaften zu entdecken.’ All dies soll uns nicht interessieren. Wenn wir das Konzept der politischan Kultur auf das Recht und seine Insciturionen zuschneiden, so wäre der entsprechende Komplex der das Recht umlagernden Einstcllungen mit "Rechtskultur" zu bezcichnen. "Wir wollen nun von der plausibel scheinenden Vermutung ausgehen, daß der Erfolg der Reformen, die im Rechtssystem der UdSSR in der letzcen Zeit stattgefunden haben bzw. noch stattfinden werden, ganz wesenclich von diesen - unsichtbaren - Einstellungen und Grundwertungen abhängt. Diese kreisen etwa um das Rechr und die Inseitutionen des Rechussystems, um die Position des einzelnen in der Gesellschaft und gegenüber dem Staat, um die soziale Schädlichkeit genauer oder ungenauer Erfüllung von Normen, um die Verwerflichkeit bzw. Akzeptabilitär krasser oder nicht krasser gesellschaftlicher Unterschiede in Stanus, Reichtum u. ä. Wir wollen den Versuch unternehmen, diese unsichtbaren Dererminanten dessen, was in der UdSSR im Augenblick vor sich geht, sichtbar zu machen: Der Terminus "vor sich gehenu beschreibr mit der zufälligen Genauigkeír der Sprache die dem Betrachter nicht offenkundige Eigengeseczlichkeit des Prozesses.

Unser Versuch der Sichtbarmachung soll dabei in drei großen Schritten vor sich gehen. Zunächsı werde ich ganz kurz das Material und die Methoden darstellen, mir denen wir arbeiten werden sowie die bisherigen Reformen und Reformvorhaben. Unser zweiter Schritt soll sich mit der Selbstrvahrnehmung und Selbstdarstellung der Juristen in der UdSSR beschätigen: Welche Schwächen, welche Stärken hat das Rechtssystem, haben die juristischen Institutionen, hat der Juristenstand aus sowjetischer Sicht? Was will man weshalb ändern, wie soll das Neue aussehen? In einem dritren Schrict schließlich wollen wir nach den alten Selbstverständlichkeiten, nach der Koncinuität der Grundwereungen und der Symbolik schauen, nach den Reflexen des Gestem in Heute sozusagen, über die die Muskeln gesellschaftlicher Steverung keine Kontrolle haben.'s

\section{Das verwendete Material}

Die folgenden Ausführungen beruhen im wesenclichen auf Interviews. Zum einen handelt es sich um die Begleitpublizistik zur - juristischen und sonstigen - Umbaupolitik von Gorbačev in der Tagespresse, in den Zeitungen "Pravdax und "Izvestijaw. In beiden Zeitungen erscheinen (seit Gorbačev vermehrr) Arrikel mit juristrschen Themen. Das Spekcrum rangiert hier von der Gerichuspublizistik zu ganz allgemeinen Beschreibungen von Mängeln im Rechtssystem und den Wegen ihrer Behebung, ganz abgesehen von den in der UdSSR üblichen Stellungnahmen von Bürgern im Rahmen der den wichtigen Gesetzesvorhaben vorausgehenden wallge-

3) Wobe der Ansarz der gesellschafulichen Codes dann nichı unbedingz uneer der Überschrift „Poliusche Kuluur a läufe und im übrigen hier auch mehrure Konzeptonen gescllschafilicher Codes zu unterscheıden sınd; zu dem ganzen s. die Kurzzusammenfassung bei A. Blankenagel, Tradition und Verfassung, 1987, 371 ., m. w. N.

4 Dic Abgrenzung zwoschen politıscher Kuleur und Recheskuliur läBe sıch naürlich necht analyusch scharl durchführen, do dic politischen Insurubonen inmer zuglesch auch recheliche Institutionen sind. Zum Begriff der Rechaskultur s. um übrigen, aus sowjerischer Sich, A. P. Semelko, Die Recheskuluar der sozialisbischen Gesellschuft: Wesen und Bestummung, in: Pravovedente 1987/4/3f. (russ.) m.w. N.: Frsilich geht es hier nichi um eine empirische Etrassung des Phinomens, sondern um telauv uninteres. sanic theorctische Konzeptioncn.

S Diese Kanirolle kann immer auch nur begrenzt scin. wcil Geschichec und Tradiuon sich niche bcliebig wegdrängen oder neutralisieren lassin, s. dazu Blankenagel, Tradiuon (Anm. 3), sowie E. Shul, Tradition, 1981 . 
meinen Volksaussprachea über diese Projekte. ${ }^{6}$ Ausgewählt wurden vor allem

Incerviewserien, d. h. regelmäßig erscheinende Interviews mit wechselnden Themen und Gesprächspartnern, aber im Regelfall gleichem Interviewer.' Darüber hinaus wurden noch sporadisch erscheinende andere lnterviews, regelmäßig mit hochgesteliten sowjetischen Juristen, sowie anläßlich besonderer Ereignisse erscheinende, programmatische Artikel und eine populärwissenschaftliche Darstellung aus rechtswissenschafticher Sicht verwertet. ${ }^{8}$ Das methodische Vorgehen folgt dem Konzept der qualitativen Inhaltsanalyse. 9

Zum anderen handelt es sich um Interviews, die ich selbst während eines sechswöchigen Aufenthales in Moskau im lezzen Herbst durchgeführe habe. Interviervpartner waren sowjetische Juristen aus sämclichen juristischen Berulssparten; auch statusmäßig repräsentierten die Gesprächspartner ein relativ ausgewogenes Bild, mit einem kleinen Übergewicht im oberen Bereich. ${ }^{10} \mathrm{Da}$ die von uns gesuchten latenten Einstellungen und Selbstverständlichkeiten nicht direkt abgefragt werden können (schon gar nicht von einem Westler in der UdSSR), bestand das Ziel der Interviews

6 Als Bespsel s. etwa Pravda vam 28.8. 1987. S. I (zur Rolle der Prokuratur/Leitarikel); Pravda vom 25.7 .1987 , S. 3 (Umweleschuez); Pravda vorn 6.7.1987. S. 3 (Unregelnäßigkeiten bei der KPdSU in Baschkunen); Izvestija vom 12.11.1987 (veralicte Normen Im Arbercsecht); lzvestija vom 9.9. 1987 (Jurssuscher Dienst der Izvestija/Fragen zum Gesezz iber dic udividuelle Arbensiaugken) usw.

7 Pravda: "Bürger. Gesellschaft. Gescez" sowic "Das Rechessyscem. W/eg des Umbausa. Izucrija:

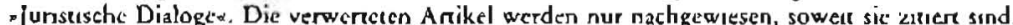
17vestijaljurisizsche Dialoge: G. Z. Araskin, 25.9.85; ders., 26. 11.1986; L. Baranov, 13.5. 1988; R. Beikın. 13.11.1986; A. Boreckij, 24.4.1987: 1. Butko, 7.9.1985; V.M.Garperen, 15.8.1986; V.T.Gubarev, 26.4. 1986; A. Gulpev, 22. 5. 1987; V P Ciribanov, 18.12.1985; V. Demin, 7. 1. 1987; S. Ivanov, 19. 1. 1986; B. B. Kraveos, 29.8.1985; I.I. Karpec, 5.4. 1985; V.L. Krylov, 26.6. 198 f; ders./I. P. Petru. chin, 30. ro. 1985; V.N. Kudnavicer, 27.8.198\%; ders., 4. 10.1985: O. Kulafin, 13.11.1987; V. V. Laxarev, 24.1.1996; A.M.Laru, 27.2.1987; N. Makarova, 17.6.1987: E.A. Lukasova, 24.1.1985i V.V.Najdenor, 29.5.1985; ders., 1.8.1986; ders., 26.11.1986; V.1. Olemik, 30. 1.1986; ders. 19.6. 1985; V. Perak, 18.2.1986; 1. Kazmir/A. Ptgolkın. 17.10.1986; V. G. Novikov, 9.8. 1985; B. Pu. gunskij, 13.2.1988; A.M. Rekunkov, 3.12.1987; V.M. Savickij, 21.3.1985; ders, 9.4.1987; ders., 26. 3. 1986; ders., 4.12.1985; /u. Scberrn, 28, ro. 1987; E. A. Smolencev, 25.4. 1986; V. M. Kogan/O. Sokol'skij, r6.9. 1987: S. Solov'eva, 29.7.1987; B. Sirisusn, 10.1. 1997: A. Sucharev, 27.2.1985; Ju. A. Tichamurov. 3.1. 1986: ders., 26. 3.1987; R. G. Tichamirov, 6.7.1986; S. Jani/V. Syrych, 26. 1. 1988; A. A. Cholavienko, 23.8.1985; G.T. Tamavskij, 16.5.1986; V.I. Terebilov, 25-10. 1986; A. M. Fila1ov, 12.6. 1989 . Repliken: 1. 12.1987/V. Andrev; $23.12 .1987 / A$. Kondratcik/A. Kallisiov; 15.7.1997/A. B. Sachidaev/A. M. Lann; 24.12.1986/C. Kac/A. Nazarenko/B. Malikov; 19.4.1987/B. Piskarev. Zwei Intervicws werden nach dem Sammelband von Ju. Fcofenov, Jur. Dialoge, 1997 (russ.), ziticr:

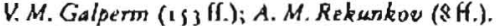

8 Pravda: 14.2.1987/Leilarikel: Stal und Persönlichkell: 6.6.1988/M.P. Vyšinskuj; 13.5.1987/ B. V. Kraucon; 25.3.1987/A. M. Rekunkov, 25.9.1987/L. Sarova, 18.9.19\$7/Leimarikel: Der Umbau und die Normen des Rechts; 12. 5. 1988/Leteanikcl: Demokratte und Gesecz; 4.8. 1986/Leteprikel: Achrung gegenüber dem Gesetz; 23.6.1988/Leitarikel: Der Rechesstaat; Izvestija: 16.8.1987/Die anonymen Denunziancen - außerhalb des Gesetzes; 18.5 .1988 , 19. 5.1988 Nodurch ist der Burokrat so susk; 21.6. 19\$8/Leicartikel: Mache und Rccht; 1.6. 1988/Letearikel. Demokrausierung und rechiliche Reform: 31.5. 1988/Inecrview mit S. Solov'cua. - Dis popularwissenschafcliche Veroffentlichung ist S. S. Alekseev, Recht und Umbau, 1987 (nuss.).

g Zu diesem Ansaiz s. etwa R. Lisch/f. Knz, Grundlagen und Anwendungsgebicte der Inhalesanalysc, 1978 , bes. 44 I., 47 f , 120 I., 137f.; A. Silbermann, Sysuemacusche Inhalusanalyse, in: R. Kónig, Hrso Handbuch der empinschen Sozialforschung, Bd. 4 . 3. Aufl. 1974, 253 ff., bes. 26, ff., 273 ff., 287 ff. sowie 299 II, s. auch J. Ritsen. Inhaltsanalysc und Idcologiekrscik, 1972, bes. If 1 .

10 Die loterviewpartener kamen aus folgenden Instituuonen/Bcrufssparcn: Instilut für Slat und Recht der Akademıc der Wissenschafeen der UdSSR, Allunıons-Forschungsinsutut für Gesetzgeburn, Akadomıc der Volkswiruschaft, Unıversität Leningrad, Institut der Prokurarur, Oberstes Genche der RSFSR, Volksgenche des Moskauer Frunze-Bezirks, Moskaver Stadt-Prokuratur, Moskauer Stadi-Xolleguum der Advokaten, Exekuuvkornicer des Rates des Kicv-Gebictes in Moskav, Moskauer Gebiets-Gericht sowie wissenschaftliche Beratung der Kommission für Geseizgrbungsvorschläge beim Obersten Sowjet der UdSSR (chem. Mitglied). Die Interviews werden im folgenden Tcil anonymisier: Sic werden nach ihrer zetticben Reihenfolge durchnumener, und nur der berufliche Hincergrund des Interviewpareners wird offenbart. Einige Informationen allgencin zu den sowjeuschen juristen s. bes N.Ja. Sokolov, Sowjeasche Junsecn als sozial-professionelic Gnuppc, Sovetskoc Gosudarsivo I Pravo 1986/2/49f. (russ.); zur Repräseneacion der Jurnsten in den Zentren der Macht s. auch E. Huskey, Specialists un the Soviet Party Apparatus: Legal Professionals as Party Functionaries, in: Sovet Seudies XL (1988), 5381. 
darin, anläßlich anderer, im übrigen nichr weniger interessanter Themenbereiche solche Aussagen zu "provozieren". Methodischer Ansatz war das unstrukturierte Incerview:" als Leitfaden verwendece ich vier Themenbereiche mic jeweils einem Bündel von Fragestellungen, die freilich entsprechend der gewählten Methode nur als grobe Orientierungslinie dienten. ': Die Fragestellungen selbsc habe ich in einigen themenlosen Probegesprächen sowie als teilnehmender Beobachter an cinem wissenschaftlichen Kolloquium in Kiew ausgearbeicet." Die konkreten Themenbereiche waren das „ Gesetz über die individuelle Arbeitstätigkeit *, das „Gesetz über die gerichtliche Beschwerde gegen unrechumäßige Handlungen von Amtspersonenu sowie die Bezichung «Bürger/Recht» und das «Selbstbild der Juristen $\propto$.

Es licgt auf der Hand, daß die Verläßlichkeit beider Materialkomplexe potentiell fragwürdig ist. Früher konnte man der allgemeinen Pressezensur in der UdSSR zumindest den wissenschaftlichen Vorteil abgewinnen, daß die Veröffentichungen "harte " Fakzen darstellcen. Bei dem, was heute veröffentlicht wird, spricht alles encweder für eine weitgehende Abseinenz oder eine völlige Absenz der Zensurbehörde: Entsprechend die Fragezeichen für die in den Zeicungen vcröffentichren Interviews, besonders, da man zusätzlich vermuten kann, daß zumindest z. T. bei Intervierver und Interviewtem die äußere, nicht aber die innere Zensurbehörde weggefallen ist. Bei den von mir persönlich durchgeführten Interviews mögen zum einen jene Interviewpartner, die aus den juristischen Institurionen kamen und die ich mangels Kennenis niche persönlich aussuchen konnte, eine besondere Auslese gewesen sein. Darüber hinaus war ich als Interviewer ein zumindest möglicher Grund der Irritation und Verzerrung in unterschiedlicher Richtung, von der platten Unwahrheic bis zu einem Rausch an Rechssstaatlichkeir. ${ }^{4}$ Schließlich gehören die verwendeten Methoden in das Instrumentarium der sog. qualitativen Sozialforschung, einer relativ neuen Forschungsrichtung. "s Die - selbstverständlich quantitariv arbeitenden - Kritiker bezeichnen diesen Methodenkomplex in sehr häßlicher Weise als "unwissenscluaftich «16 - auch dies ein Beispiel gesellschaftlichen Widerstandes gegen Innovationen.

it S. etwa allgemein Bureas of Appl. Soc. Research, Das qualitative Interview, in: R. Kömg, Hrsg., Das Interview, 1962, 143f.; R. Seebbins, The Unstructured Research Intervicw as Incipiene Incerpersonal Relamonship. in: Sociology and Socia Research s6 (1971/72), 164 f.: M. Kohli, "Offencsa und sgeschlossenesi Intervies: Neue Argumente zu einer aleen Kontroverse, in: Soziale Wele 29 (1978), i f.; A. Whizel, Verfahren der qualieaciven Sozialforschung, 1982; G. Schneider, Hermencusische Sirukiumalyse von qualizauven Inrerviews, in: KZISS 40 (1988), 223 ff; allgenein s. auch noch E. Erbstob w. a., Sudien zum Interview, 1973.

12 Darüber hinaus wurde der Intervicw-Lculaden im Verlauf der Intervicws modiriziert, ebenso durch neue Schwerpunktreczung vorhandener Fragen wie auch durch Auslassung solcher Fragen, mu denen dic sowjcuschen Gesprächspartner nichts anzufangen wußten (so etwa der Frage danach, welche Literatur-Hilfsmitel man bei der junstischen Bearbetung eines Problems benutzc).

13 D2s Kolloquium bischälugic sich mu Fragen des Strafrechis und zwar dem Problem der Bcgründung des staatlichen Strafanspruches und der Öffendichkeıl des Gerıchisverfahrens: Die grundlegenden Fragen, dic bei diesen Themenkresen berühre wurden, ermoglichien dann cin schon rechi gezieles Ausarbcien des Inicrview-Letrfadens.

14 Weltere mögliche Quelle der Verzerrung waxcon dic Gesprïche selbst, da ich als Interviewer die Funkuon hatte, bestummte Punkte anzudiskutueren, aber eben niche unbedingt auszudiskuueren, was angesiches der Themen und der interessanten Ausführungen der Gespräechsparner oft niche lecche war. Mit dem Uberwechscln in die Rollc des sechren $\propto$ Gesprächspanners kam es jedach in der Diskussion mu dem Prablem des "Rechthabens/niche Rechthabens* zu wexceren Verzerrungen.

is S. etwa die Kontroverse zwischen S. Kleıng. Umriß zu ciner Mcthodologıe qualizativer Sozıallorschung, KZFSS 14 (1982), 224 l. sowic T. P. Wilson, Qualitaluve soder quantitauve Mectiode in der Sozjalfor-

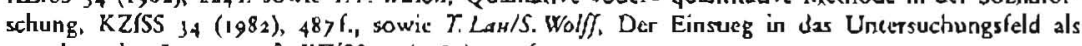
soziologischer Lemprozeß, KZISS is $\left(\mathrm{rg}_{3}\right)_{4}, 417$ f.

16 Die Kontroversc lühre dann vor allem zur Entwicklung der Edhnomethodologie, s. E. Weingarten! F. Sack/J. Schenketn, EthnomeLhodolog1e. 1976. 


\section{Reformen und Reformvorbaben}

Betrachten wir zunächst das, was in der Geseczgebung geschehen und was für die Zukunft geplant ist. Ein Schwerpunkt der schon durchgeführen Reformen liegt im Wirtschaftsrecht: Mit dem „Gesetz über die individuelle Arbeirstätigkeit», dem „Gesetz über das sozialistische Unternehmen « und, ganz frisch, dem »Gesetz über die Kooperative $\alpha$ hat die UdSSR ihr bis dato größrenteils staatliches Wirtschaftssystem auf drei unterschiedlich starke Beine gestellt und im übrigen schon existierenden privatwirschaftlichen Formen eine gesetzliche Grundlage gegeben. Erwähnenswerc sind weiter das ${ }^{2}$ Gesctz über die gerichtliche Kontrolle unrechtmäßiger Handlungen von Amspersonen "sowie das "Gesecz über die Diskussion von Gesetzesprojekcen $\alpha .{ }^{17}$ Durchgeführt wird im Augenblick die ungeheuere Arbeit der Bereinigung und Anpassung der ministerialen Instruktionen an die durch das Gesetz über das sozialistische Unternehmen veränderte rechtliche Lage in der Volkswirtschaft. Geplant ist weiter ein neues Strafgeserzbuch mit erheblich bereinigten Strafbarkeitsvorstellungen ${ }^{18}$, ein neues Strafprozeßrechc (freilich ist der Encwurf geheim - alte Gewohnheiten sterben schwer), ein Pressegesetz, ein Gesetz über die Öffentlichkeit der Staatsverwaltung, ein Gesetz über Patentc, Erfindungen und Lizenzen, und schließlich, neben einer Reihe von Verwaltungsreformgesetzen, ein Gesetz über die Normativakse, mit dem Orónung in das Chaos allgemeiner und allseitiger munterer Rechtsschöpfung gebracht werden soll." Dazu kommen noch jene z.T. sensationellen Reformen, die sich in der Konsequenz der gerade abgeschlossenen XIX. Parteikonferenz ergeben: Erste Schritte sind hier mit einer Veränderung der Verfassung und des Wahlgeserzes unternommen worden: besonders erwähnensrver die Professionalisierung des Obersten Sorvjers (OS) (zwei 3-4 Monate dauernde Sitzungsperioden), die Begründung eines neuen, nicht professionellen , Grundparlamentse, des ,Kongresses der Volksdeputierten<, die erhebliche Stutzung der Befugnisse des Präsidiums des OS, die Einrichtung eines Staatspräsidenten-Amtes mit weitgehenden, freilich an die Kontrolle des OS gebundenen Kompetenzen und schließlich die Einrichtung einer Institution der Verfassungskontrolle, des Verfassungsraces. ${ }^{20}$

17 Gesciz wiber das s0zualistusbe Untemehmen: VVS SSSR 1987/26, An. 386; ene deutsche Úberserzungs. bei G. Frunden/W. Scbreat, Das sowjerische Berriebsgesciz. Ein Vergleich von Enrwur und Endfassung (Arbeiten des Osteuropa-Insuruts Nr. 121, 1987). Gesetz über die indiruduelle Arbeustäugkeu: VVS SSSR 1986/47, Arr. 964; cinen deusschen Texe s. in: WGO 1986, 369 f.: zum Gesetz s. T. Schwesffurth, Die Komplementantat persönlichen Nutzens und gesellschaftlichen Interesses, Ostcuropa-Ruchi 1988. if. Gesetx über die Kooperattve: Ekon. Gaz. 19\$8, Nr. 24; s. dazu etwa A. Maksmovic, Die Encstehung

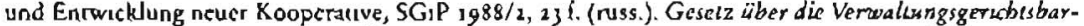
kett: VVSSSSR 1987/26, Arr. 388 ; Änderungsgeselz, un: VVS SSSR 1987/42, Arc. 692; zu dem Geselz K.J. Kuss, Gencheliche Vervaltungskontrolle in der Sowjetunion, JOR XXVIIU/2 (in87), 27If.; eunen deutschen Text s. ebd., 425 H. Gesetz, über die Volksaussprache- VVS SSSR 1987/26. An. 187 .

18 Als Vorsiudie zum neucn Sersigeseczbuch s. V. N. Kudravucev, Hesg., Das Strafgeserzbuch: Ein theoretssches Modell, Moskau, 1987 (russ.); s. jelxe dic Veróffenelichung des Entwurts in dec izvestija vom 37. 12.1988.

19 Dabes handelt es sıch z. T. um paliusche Absıchiserklärungen, z. T. um konkrete Gesetzgebungsprojekıe in Gesetzgebungsplan, s. dazu VVS SSSR 1986/37, Art. 782; erwähnenswert etwa noch die neven Einund Ausreisevorschriften. SIPP SSSR 1 1986/31, Art. 16); dic Normen zu den " jome ventures $*$, VVS SSSR $1987 / 2$. Ar. 35; SPP SS5R I 1987/9, Ar. 40.

$20 \mathrm{~S}$. den Enrowut det Veffassungsänderang in der Pravda vom 22.10.1988 und die nach lebhatier Diskussion verabschiedece endguiluge Fassung in der lzvestija vom 3.12.1988/4, 12. 1988. Das Abschlußkommunaque der Pareeikonferenz s, in der Izvestija vam 5.7. 1988. - Allgemeun zu den Ändcrungen im Rechissystem s. noch $K$. Westen, Perestrojka und Rechisordnung, Ostcuropa 1988, 329 ff., sowic

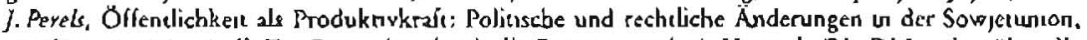
In: KJ 20 (1987). 183 ff. Zur Rezeprion durch die Presse s. auch A. Hasernch, Die Diskussion über dic 'perestrojka. in der sow/etuschen Rechisplege. Ostcuropa-Recht 1988, $20 \mathrm{~g}$. 
Welche Schwächen konsurieren nun unsere sowjetischen Gesprächspartner in ihrem eigenen Rechtssysıem. ${ }^{21}$ Wenden wir uns beispielhaft zunächst dem Öffentichen Recht und dem Wirtschaftsrecht zu, zwei in einem staatswirtschaftlichen System eng verbundenen Rechtsgebieten. Die im Folgenden verwendere Darstellungsmethode besteht ausschließlich in wörtlichen oder sinngcmäßen Zicaten sowjetischer Autoren: Die Selbsrwahrnehmung ist also so authentisch wie möglich.

In erster Linie ist man mit dem Normensystem als solchem unzufrieden. Die Verfassung soll endlich unmictelbar geicendes Recht werden; die falsche Vorstellung, daß eine Verfassungsnorm (ebenso wie auch ein Gesetz im formellen Sinne) zu ihrer Wirksamkeit eines konkrecisierenden untergeserzlichen Akres bedarf, muß aufgegeben werden, ebenso wie die Vorsrellung, die Verfassung wolle nur das absichern, was sic zum Zeitpunke ihres Erlasses vorgefunden habe. ${ }^{22}$ Auch bei der sonstigen Rechtsschöpfung herrschen katastrophale Zustände. Viele Normen erschöpfen sich im Deklarativen und stehen nur auf dem Papier; die Durchsetzung der Norminhalte ist nicht durch Sanktionen gesichert und entsprechend laufen die Normen Icer. ${ }^{23}$ In der gesellschaftlichen Wahmehmung herrsche hier eine Schizophrenie: Einerseits sei man $\operatorname{der}$ Ansichs, $d_{a} B$ es genüge, gute Gesetze zu machen. Andererseics sei der Erlaß eines Gesetzes eine unbedeurende Angelegenheit: Man warte auf den Erlaß des unrergesetzlichen Aktes oder der ministerialen Inscruktion, dic dann dem Gesezz seinen eigentichen Sinn und seine Geltungskraft geben. ${ }^{24}$ Sehr anschaulich spiegelt sich das in folgendem Ausspruch: In einem Leserbrief anläßlich des heißdiskutierten Problems der Bestechung schricb eine Leserin, man solle doch nun endlich eine entsprechende Vcrordnung erlassen, damit das Bestechungsunwesen sein Ende habe. ${ }^{25}$ Freilich sieht die Sicuation bei den untergeseczlichen Richtlinien und Instruktionen auch nicht besser aus. Da jedes Ministerium - und die Zahh der Ministerien in Union und Republiken ist sehr groß - zu allen ihm wichrig erscheinenden Punkten eine Instruktion (ecwa für die unterstcllcen Wirtschaftseinheiten) herausgibr, herrsche ein einziger Normenwirrwarr. ${ }^{26}$ Die Instruktionen werden nicht einheitlich veröffentlicht, und die Geltungsansprüche der Instruktionen gegeneinander sind weder in zeitlicher noch in hierarchischer Sicht geklärt." In der Praxis ist nur klar, daß die Instruktionen gegenüber den ibnen übergeordneten Gesetzen durchschlagende Geltung haben und daß sich die Verwaltung im übrigen,

21 Ein Blick auf dic sozıale/instrtutsonelle Zusammensetzung seı gewagt, dic con Indikator für den EinfluB uneerschiodlicher Junstengnuppen seın mag. Bes esner Einstellung nach den Xategonen Wissenschale (29)/Genchte (6)/Prokuratur und Ermulungsbehörden (22)/Venvalung mit Justuzmunisterium (9) und sonsugen (1) zeige sich, neben eines Wissensehafuslastigkeit, cin spezifisches Übergcunche von Prokuraur und Emutelungsbehorden gegenuber den junstischen Insututuonen. Dic Mengenvereilung bet den von mur durchgefuhren Intemews beurug in der Reihenfolge $9 / 3 / 2 / 2 / 2$. Die Wissenschaltslastigkeit dürfie im ubngern wenger reale Machı widerspiegclo als vielmehr dic durch uneerschiedliche Faktoren bedingu: Autrakivilät von Gesprächsparnem aus dem Bcretch der Wissenschalt. Zur Zimerweise: Die Nachwelse sind nur exemplarsch für mehrere/viele solcher Äußerungen, sowert nuche wörlich zutere wird. Die Interviews aus den Zetungen weeden mit dem Namen des Interviewten zluer; bei Mehrfachintcrvicws der gleichen Person ourd zur Klarstellung das Dalum der Zütung honugeselzl. Die Lettarikel werden per Titel zitıer. Zur Zilterweise der von mur durchgelühren lnterview's. Anm. 10.

22 Sevickij (Anm. 7/9.4. 1987); Lazarev (Anm. 7); Interview y/Wiss.: Konksel gng is dabes cawa um An. 59 A bs. 2 Verl. UdSSR, das Verfassungsgebol oder Nichigebor der Einnchtung ciner Verwaltungsgerichubarkell.

23 Solov'eva (Anm. 7): Umbau und Normen des Rechis (Anm.8).

${ }_{24}$ Tichomyou (Anm. 7/3.1.1986); Rekurkov (Anm. 8); Choljavconko (Anm. 7); Aleksece, Reche (Anm. 8), 131.

25 Im lnecruew 4 Nhiss. gegenúber dem Verf, ziuer.

I6 Lazarre (Anm. 7); Choljavienko (Anm. 7); Tichomiros (Anm. 7/26.3.1987).

27 Solov'cea (Anm. S): Kntik an gesetzesändernden Instruknonen; Choljavëenko (Anm. 7); Interview 1) Wiss. 
weist man sie auf den Vorrang des Geserzes hin, um solche Hinweise herzlich wenig kümmert. ${ }^{28} \mathrm{Da}$ diese Instruktionen ebenso jegliche Kreativität wie auch jedes wirtschaftlich vertretbare und sinnvolle Handeln verunmöglichen, führen sie selbst im durchaus nicht kostenberußt arbeitenden sowjetischen Wirtschaftssystem zu verbreiteten Ausweichmanövern: ${ }^{99}$ Die automatische Gesetzes- und Instruktionsumgehung wird zur Gewohnheit und ist feste Tradition ${ }^{30}$, und dieser Wcg linksherum (Originalausdruck) differenziert dann nacürlich nicht mehr zwischen vernünf́rigen und unvernünftigen Normen. Eine steigende Tendenz har folglich auch die Nachfrage nach guten Juristen in der Wirtschaft: Wenn schon ein Ausreizen und Umgehen von Vorschriften, dann möglichst ein juristisch abgesichertes." "'

Weitcrer Brennpunkı allgemeiner Kritik ist das Rätesystem in seiner heutigen Gestalt, die den ursprünglichen Bauplan geradezu auf den Kopf scellt und die gesamte Konzeption auf allen Stufen der Rärc bis hin zum Obersten Sowjec leerlaufen läßc. Theoretisch ist der Rat alles und das Exekutivkomitee ein von ihm gesceuertes Umsetzungsorgan ${ }^{32}$; praktisch ist das Exekutivkomitee der Hund, der mit seinem Schwanz (dem Rat) fröhlich gegen allerlei hohle Gegenstände schlägt und sich so Akklamacion holt." Die Gründe sicht man ebenso in der russischen Tradicion des Übergewichts der Exekutive wie in den kurzen Tagungsperioden und der mangelnden Professionalisierung der Deputienen wie auch in den falschen Selekrionsmechanismen. Die nützlichen und wertvollen Mirglieder der Gesellschaft, die normalerweise in den Räten sitzen, sind, so hat man erkannt, nicht immer unbedingt jene, die auch für die Arbeit in den Repräsentativorganen geeignet sind. ${ }^{34}$ Kritisier wird schließlich, in leczter Zeit zunehmend, die Verquickung von Staat und Partei sowie das Fehlen jeglicher Inkompacibilitäten und auch die zeitlich nicht bcgrenzte Innchabung von Ämuem. 35

\section{Strafrecht}

Betrachten wir als einen zweiten Bereich das Strafrecht mit den Augen der sowjet;schen Juristen. Ähnlich wie das laienhafte Verständris von Recht, das dieses auch immer mit Strafrecht identifizier, war das sowjerische Rechesdenken durch eine Überbetonung des Strafrechts gekennzeichnet. ${ }^{6}$ Mit zunchmender Differenzicrheit des Bildes vom Recht möchte man diese Gewichtungen zurecht- und das

28 Wobei der Terminus des Voreangs des Gesezxes Irüher in der sowjeuschen Rechesdogmatik und Recheswirklichkeı orcht exıstierte, man sprach vielmehr von ,höchsecr junstıscher Kriftu. Offiziell engefühn wurde der Terminus ersi durch das Abschlußdokument der XIX. Paneikonícrenz (Anm. 20). Zur stärkeren Geltungskraft der instrukıonen s. e(wa Boreckij (Anm.7), Clooljavicenko (Anm. 7); Lukajova (Anm. 7), lntervicw 12/Wiss.; Inservicw 2/Wiss. Für enc stärkere Einhaltung des Narm huer auch Alekserv, Reche (Anm. 8), S1, 98: lückenlose Gesctzgebung zur Ausschaleung van Instrukuonen.

29 Rekunkor (Anm. 8); s. freilich Filawos (Anm. 7), zur Möglichkett gesetzestreuen und winschaftlichen Handclns.

30 Tuchomrov (Anm. 7/26. 3. 1987): Naldenow (Anm. 7/29.5. 1985), mit Ziffern und Besspielen, dic freilich auch un anderen Winschaftxsysecrnco als nicht umgehbar lyngenommen werden (unbefuguer Gebrauch

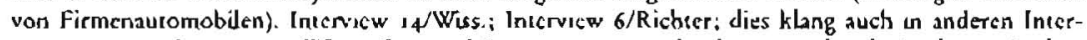
views an, erwa Ineersick' 4 /Wiss.: Giscez: könnc man ummor durch entsprecliende Auslegung/andere Gesetze umgchen. Damiı mag sich fredich auch dic manchmal bedrückendc Beliebigkeı kunstregelrocht begründeter junstischer Entscheidungen dokumenucren, der jeder Jurise un seincr Bcrufspraxis kontınuerlich ausgeserse ist.

11 Interview 6/Richter (frcilich ohne diesc Abstcht gut zu heißen); Interview i 8 NWiss.

32 Solov'eua (Anm. 8).

33 Kueafin (Anm. 7).

34 Kulafin (Anm. 7); Solov'eva (Anm. 8).

3s S. wioderum das Abschlußdokument der XIX. Parteikonfcrenz (Anm. 20) sowie Kritafir (Anm. 7); Solov'rua (Anm. 8). Die Zeitgrenze nach der Verfassungsänderung liegr ba 10 Jahren.

36 Alekseev (Anro.8), 22; Sarkkij (Anm. 7/4.12.198/); zur Kreik s. auch Larm (Anm.7/19.7.19\$7); Piskarev (Anm, 7). 
Strafrecht ein wenig in den Hincergrund rücken. Darüber hinaus konstatiert man nun da man endlich dic rosasozialistische Brille abgenommen hat -, daß ebenso die bisherige Strafrechtspolicik wic auch das Steafrecht als Instrument sozialer Kontrolle völlig ausgehöhlr sind und daß die alten Weisheiren von der in diesem Falle wirklich urwüchsigen Kraft sozialistischer, aber nicht planmäßiger Gesellschaftsentwicklung in den Boden gestampft worden sind. ${ }^{77}$ Strenge Sanktionen, hohe Strafen, eine allgemeine Straineigung und auch zweifelhafte Beweistechniken - das Geständnis etwa als König der Bewcismittel - hießen die bisherigen Leitlinien. ${ }^{18} \mathrm{D}_{2}$ Resultat dieser archaischen Strafrechespolitik sind niclit überraschend auch archaische gesellschaftliche Zustände: Die Gesellschaft funktioniert ganz wesentlich über einen allgemeinen Kreislauf von Geschenken - juristisch und weniger freundlich nennt man dies Bestechung!'9 Das Staatseigentum hat ein wenig vorzeitig den Zustand des allgemeinen Gemeinschaftseigentums erreicht. Im Produkeionsbereich und im Konsumbereich herrscht das Prinzip allgemeiner Selbstbedienung ohne irgendwelches Unrechtsbewußrsein: »lm Handel stehlen alle», so cine Beschreibung mit jener Selbscverständlichkeit, die man sonst nur für das Wetter aufbringt. ${ }^{\circ}$ Schließlich gibt es auch organisiertes Verbrechen in den uns bekannten Bereichen Prostitution und Drogenhandel; daß jedoch auch Unionsrepubliken bzw. Ministerien den Zentralscaat mit nie existencen, aber hoch prämierten Baumivollrekordernten jahrelang betrogen haben, da ist die UdSSR uns nun doch schon weit voraus; Zitar: Immerhin wurde so klar, weshalb es immer mehr Baumwolle und immer weniger Baumwollprodukte gab."1

\section{Die Institusionen}

Verlassen wir nun die beispielhaft ausgewählzen Rechesgebiete und werfen wir wieder durch die Augen der sowjetischen Juristen - einen Blick auf die Institutionen. Im Kreuzfeuer der Kritik stchen hier vor allem Gerichte, Prokuratur und Ermittlungsbehörden. Das Heil der Zukunft wird in einer Verstärkung der Rolle der Gerichte und auch der Anwaltschaft gesuche. ${ }^{2}$ Kritisiert wird die schlechte Arbeir sowohl der Ermitulungsbehörden wie auch der Prokuranr und der Gerichte in Strafsachen: Die Ermittlungsbehörden und auch dic Prokurarur in ihrer Rolle als Staatsanwaltschaft dominierten die Gerichte. Diese hätten vor beiden Vertretern der Anklage Angst, ja befänden sich in einem geradezu hypnotisierten Zustand und schlössen sich mehr oder weniger blind der Anklage an, wie sich an der geringen Zahl von Freisprüchen und an der noch geringeren Zahl von erfolgreichen Berufungen und Revisionen ablcsen lasse. ${ }^{13}$ Sei wirklich einmal die Anklage durch den Gang der Hauptverhandlung widerlegt, so hätten die Strafrichter nicht etwa den Mut zum

37 S. die Kntrik der Ineffizenz des Serafreches beı Kudrjavcev (Anm. 7/27.8. 1987); s. auch dic Nachweise in Fn. 23.

38 Sébenn (Anm. 7): al gemeine Stralneıgung: Savickij (Anm. 7/26. 3. 1986): schlechec Verzendigungsmög. lichkeiten: ders. (Anm. 7/4,12.1985): auch Hene Frciheisstrafen als schwerwiegend; Makarova (Anrn. 7): Incerview 1/Wiss.

19 Olejuk (Anm.7/10.5. 1986); lnterview 2 NWiss.

40 S. vor allem Olejnik (Anm. 7/30. 5. 19\$6); 5. eld. auch das Internew mit der Sirafgelangenen Garına. 41 Pravda vom 23. 1. 1988, S. 3.

42 Kudryaveev (Anm,7/27.8.1987), ders. (Anm.7/4.10.1985); Smolencev (Anm. 7); Aleksecu, Reche (Anm. 8), 141, 148; Sachidaev/Larnn (Anm, 7); Kravcov (Anm. 8): Terebilov (Anm. 7); s. auch Petruchum (Anm. 7), 3,u Gründen gerichtlicher Fehler und der Rolle der Advokatur beı der Aufklärung solcher Rechisprechungsfehler. S. im übrigen Kudrjaeces (Anm. 7/27.8.1987) sowie Krylov (Anm. 7), zum verfassungsmäßigen Status und schlechten Ansehen der Advokator; in gleicher Richtung Interview 7' Advokat.

43 Seberm (Anm. 7): \{akrısche Dominuerung des Gerichus durch Ermıclungsbehörden; Saeuckij (Anm. 7) 21.3.1985); Smolencev (Anm.7); Kravcov (Anm. 8); Guljarv, Terebulov (beade Anm. 7), für einc Trennung von Prokuratur und Ermutulungsbehörden. 
Freispruch; die Angelegenheit werde dann vielmehe zur zusäczlichen Nachermitrlung an die Ermittlungsbebörden zurückgegeben. ${ }^{\text {th }} \mathrm{Im}$ Ergebnis komme es bei allen drei Inscitutionen zur bekannten Strafneigung und zu den eraühnten hohen Verurteilungsquoten. Das habe freilich noch andere, systemische Gründe, die es zusäzzlich abzustellen gelte. Die Arbeit all dieser juristischen Institutionen werde über ein Syscem von Planziffern/Leistungsindikatoren beurteit, das jeweils Anklage und Verurteilung lohnender mache als Freispruch und Einstellung."s Die Praxis has sich diesen Vorgaben angepaßt: Geleiter von dem Bestreben, einerseits auf hohe Leiszungszahlen zu kommen und andererseits die Negativa - die für den Bürger günstigen Entscheidungen - so um die Bilanzen herumzumauscheln, daß sie dort keine Negacivposten bilden, klärt man alles auf, klagt man alles an und verurteilt man nach Möglichkeir. ${ }^{* 6}$

Noch schlimmer steht es um die Unabhängigkeit und das Ansehen vor allern der Gerichte. Mit der Unabhängigkeit, die immerhin verfassungsrechtlich garanciert ist, ist es nach allgemeiner Ansicht nicht weit her. Dies liegt nur begrenzt an der Wählbarkeit der Richteri7; Einflußnahmen von außen sind hier auf Empfehlungen zur Wiederwahl durch die örtliche Partei- und Exekutivspitze beschränkt. Dic materielle Siruacion der Richter, ihre sachliche Unabhängigkeit, ist vielmehr so ungesichert, daß die örtlichen Organe der Macht im Bedarfsfall immer ein Druckmittel in der Hand haben: Wohnung, Fahrzeug, die Zurverfügungstellung von Arbeitsmitteln, die Instandhaltung der Gerichtsgebäude usw. sind eine Gabe, die die örtliche Exekurive gewähren oder auch nichr gewähren kann. ${ }^{4}$ Solche Abhängigkeiten führen dann zusätzlich noch zu vorauseilendem Gehorsam - angesichts der Gegenleistung der Exekuive freilich vergebens. Der Durchschnitt der Gerichrsgebäude rangiert zwischen Baracke, Hinterhof und halbzerfallener Ruine ${ }^{49}$; keine Rede kann sein von den "Palästen der Gerichtsbarkeit», die sich manche Autoren wünschen und in denen dann hochbezahlte Richter mit vorzüglicher materieller Ausstattung und hoher professioneller Qualifikation sowie auch dem für den Beruf des Richters unerläßlichem Quantum an Lebenserfahrung (trau keinem unrer 30 ) in unabhängiger, freilich screng an das Gesetz gebundener Rechesprechung die Gerechtigkeirsvorstellungen der sozialistischen Gesellschaft formulieren und verwirklichen - ist doch der Richter, wie unisono vertreten wird, das eigentlich demokratische Justizorgan. ${ }^{50}$ (Ganz offenkundig zieht die Lokomorive des Umbaus auch einige mit parcikularen Gruppeninceressen beladene Waggons.) Vorläufig aber sieht es noch ganz anders aus: Sein sozialer Status im Rechtssyscem ist so niedrig, daß ein Wechsel in die Prokuratur von der Bevölkerung als Beförderung wahrgenommen wird, bzw., noch drastischer: Im komplizierten Mechanismus des sowjecischen Reches ist der Richter ein Mensch zwischen Humanismus, Gesetzlichkeit und Telephon (Zitar)."

44 Krylov (Anm. 7); Smolences (Anm. 7); Culjaev (Anm. 7), für die Abschaifung des zusärzlichen Ermit̨lungsverfahren: Sarickij (Anm. 7/4, 12.1985).

t5 S. dazu die ausfuhrliche Darscellung bei Saurskij (Anm. 7/4.12.1985): Kondrąčk/Kalliseov (Anm. 7).

46 S. Kravarou (Anm. 8), zu den Verfissungsprinzipien der Genehesbarkest.

47 Terebiloo (Anm-y): Hauptproblem der Genchtsbarkete sei dic Reform der Unabhingigkeır; Alrkseev. Recht (Anm. 8), 148: deo: s. derngegenüber die wake Linte bei Kravcov (Anm. 8): Beisplele lür neheerliches Fehlvechalem und andererseses richeerliche Unabliängıgkeıt als Frage persönlicher Qualicaren und nicht instıtuenoller Absicherung.

4 S. etwa Kogan/Sokol'skıj sowie Kac/Nazarcnko/Malkkow (Anm. 7); Intcrvicw 16NWLss; Intervicw 18/ Wiss.: auch huer dic Beconung der Richecrpersönlichkert.

49 Maksrosa (Anm. 7).

so Sucharev (Anm.7); Kudnaveres (Anm.7/4.10.1989): ders. (Anm.7/27.8.1987): Haus des Reches; Alckseev, Reche (Anm. 8).

51 Interview 6/Richter; Interview 12/Wiss.: Das bedeutce frcilich nicht, då dic Lage in anderen Instıtutı- 
Nichr besonders gut machr sich auch aus den Augen sowjetischer Juristen dic reale Bezichung des Bürgers zum Rechtssystem. Beklagt wird eine niedrige Rechtskultur (im wertenden Sinne) und eine niedrige bis katastrophale Rechtskenntnis sowie ein nur schr schwaches Vertrauen in die Arbeit aller juristischen Institutionen, von den Gerichten über dic Prokuracur bis hin zur sowieso nur geringschätzig betrachteten Anwaltschaft." Letztlich habe der einfache Bürger überhaupt keine Vorstellung vom Funktionieren des Rechtssystems. Das Recht werde generell damit idencifizien, daß etwas verboten sei. Das Volk betrachte die Gebove der Rechtsnormen mehr als eine An Empfehlung. Sei dies Gebor/Verbot der Rechisnorm unsinnig und unzweckmäßig, so werde es eben umgangen und mißachter. ${ }^{\text {s }}$

Als Folge des Scalinismus herrsche überall noch ein Klima der Angst oder zumindest des Mißtrauens gegenüber dem Recht:"4 Allgemein sei die Tendenz zur Unterordnung und eine völlige Teilnahmslosigkeit; Beamte hätten demgegenüber das Selbstgefühl eincs Zaren. Akriviät äußere sich nur in einer allgemeinen Bcschwerdewur aller Bürger. Hervorgerufen werde diese durch die eigentümliche Vorscellung, daß ein gesellschafclich nüzzlicher Mensch Beschwerden schreibe:ss Deren Speerspirze wiederum is die allseits beklagte Schar der anonymen, of professionellen Denunzianten. ${ }^{66}$ Charakteristisch für die Beziehung des Bürgers zum Recht sind weiterhin archaisch strenge Vorstellungen über den Sinn und das Maß von Strafen. 37 Verbreitet ist auch die gesellschaftliche Tendenz, Konflike unter Umgehung des Rechtssystems beizulegen; sie entspricht einer noch aus dem zaristischen Rußland kommenden Doppelgleisigkeit in der Wahmehmung des States: ungeheure Wichrigkeit und große Rolle einerseits, völlige Bedeurungslosigkeit für das persönliche Leben andererseits. ${ }^{8}$ Dieser *juristische Analphabetismus und "juristische Nihilismus habe eine lange, wieder russische und sowjetische Tradition: Die schon bei Dostojewskuj zu findende kulturelle Mißachtung und Abwerrung des Rechs habe sich bruchlos über marxistisch-rheorecische Absterbetheorien und den Stalinismus bis in die jüngste Vergangenheit der Erstarrung fortgesetzt. 9 Woher solle der Bürger auch

nen nuche auch knessiert worden wäre, 5. Fïr die Prokuratur e(wa Guljaev (Anm. 7); Kogan/Sokol'skij (Anm. 7); Kudnaucev (Anm. 7/27.8. 1987): Pukavev (Anm. 7): KarlNaxarenko/Malhkov (Anm, 7)i s. auch Baranov (Anm, 7) allgeman zu den Denkmustem in der Jusaz.

s2 Gubayev (Anm. 7); Aleksecv, Recht (Anm. 8), is8; Interview 12/Wiss.: Genche als Insurutionen der Strafe.

33 Intervicw I2/Wiss.; Intervicw is/Richter; s. 1. übr. dic Nachwesse bei Fri. 22 f. 2ur Normenumgehung im Wireschalessysecm.

54 Interview s Wiss.; Intervicw 16/Wiss; lntervew 8/Richicr; Intcrvew 9/Prokurarur: Demgegenüber uucht dann aber immer wieder der y.Mythos des mutigen Russena avf, х. T. beı Gesprächspannern, die vorher das Phänomen der Angst bejaht hateen: cwa Interview ro/W/iss.; Interview il/Prokumar

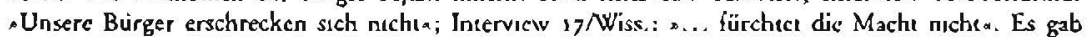
I. übr. auch Gesprächspanner, dic das Phänomen der Angse grundsätzlich in Abrede stellecn, etwa Ineerview is/Richter: Daber bleibt natürlich dic Frage, unwewent die Antworten ehrlich waren. - S. schließlich noch dic cewas eigenarrigen Zukunfisvisionen bel Alekseev, Recbe (Anm.8), 172 some Rekunkov (Anm. 8); Demokratie und Geset2 (Anm. \$) - bexde zur Rechisunternchtung und Rechespropaganda uneer den Bürgern.

is Intervew in Wiss.; Interview $3 / W_{1}$ iss.: Beschwendeführer als persönlicher Fend der Verwalung; Interview 8/Richcer; Interview 10 Wiss. - fast unmucelbar im Anschluß an die Aussage, das Yolk habe Angsr; Intervic w ${ }_{12} /$ Wiss.

s6 Saurkij (Anm. 7/21. 3. 1985); Inierview s/Wiss.; Interview ו1/Prokumtur; Interview 17/Wiss.: Pnnzıp der straflasen Anschuldigung als Erbschalt von Stalin; Lectarikel $n$ Den anonymen Denunzianten außerhalb des Geserzes* (Anm. 8)

57 So erwa Intervico 10 NWiss.

58 Interview $14 /$ W/iss.; Interview 9/Prokuratur: Interview 2/W/iss.: Gerichtc als Organ gescllschaftlicher Repression; Interview i NWiss.: Kein Glaube an die Mache des Geveizes, weil der Staas sich selbse nichı an dic Gescess halle.

19 Intervicw 2 WWiss.; Intervicw 3/Wiss.; Intervicw 7/Advokacur; Incervew i6 Wiss. 
eine gute Beziehung zum Recht haben, wenn völlig ungeachter der Rechtc, die ihm die Rechtsordnung gebe, er überall nichts weiter sei als ein kleiner Bitusteller. ${ }^{\infty}$

Ganz anders wird demgegenüber die erwünschte Beziehung des Bürgers zum Recht definiert: Kenntnis der Rechtsnormen, angstfrei, ein wenig, wenn auch nicht zu kritisch, Geseczesgehorsam oder auch strengstc Beachtung der Gesetze wurden hier genannt. ${ }^{\sigma_{1}}$ Das häufigste Auribut war freilich die "Achrung vor dem Gesctz, bczeichnet mit jenem Terminus respektvoller Anerkennung, der mit dem Ausspruch "Du achtest mich niche die schon ritualisierte Eingangsformel von Streitigkeiten und Schlägereien unter Betrunkenen ist (zumindest meiner, in diesem Fall nicht teilnehmenden, Beobachtung nach).

\section{Der Abschied von aleen Symbalen}

Gesellschaftlicher Wandel vollzieht sich immer auch auf der Ebene der Symbole: Die kennzeichnenden Verdichtungen der alren gesellschafelichen Ordnung weichen den neuen, sei es in der Folge gesellschaftlicher Veränderungen, sei es simultan mit ihnen oder sei es auch ihnen vorauseilend.62 In einem Staat wie der UdSSR, in dem die Selbstdarstellung der Macht, in dem Schlagwore, Rituale und Symbole einc ungeheuere Rolle spielen ${ }^{6}$, läßt der Versuch doch erbeblichen gesellschaftlichen Wandels auch spektakuläre Symbolauswechshungen erwarten. Wir werden nicht entüuscht. Telefonrecht, administrativc Befehlsmethoden, Gleichmacherei, Papierschöpfung, Behördenscheuklappen, Augenwischerei, Vorzeigetum, Lokalismus, Kampanientum, Bürokratismus und anderes mehr bezeichnen die negative Vergangenheit, die "Zeit der Erstarrung". Signifikant gering im übrigen das namedropping der Klassiker. ${ }^{6}$ Die alte Toleranz gegenüber dem Alkoholismus wird abgelegt (leider); aus dem Verteidiger der Revolution Felix Dzeržinskij wird ein Geheimdiensimann mit blurigen Händen; die Rechtsstaatlichkeit der Strafverfolgung wird zum Maß des Wertes des Rechtssystems; dem Freispruch, nicht der Verurteilung, wird erzieherischer Wert bcigemessen; das Strafrecht verliert unter den Rechrsgebieten den Status des aprimus inter pares ${ }^{\text {bs }}$ Ansonsten ist alles

60 Zum Bürger als Bıtcsıeller s. Baranov (Anm. 7); s. demgegenüber die Visıon Alekseevs, Rechı (A nm. 8), ir2: Ein Bürger, dessen Rechte kcine Gabe des Staates mehr sind. Intervico 3NWiss: automatusche Tendenz der Verwaltung, alles zu verbieten.

61 Interessant war hier zum einen die immer wieder aultauchende Apostrophicrung der Bezuehung des Bürgers zum Gesetz (oder auch besummter Verhaltenswersen) als ngesund/kranku (s. a. Anm. 83 ), enc Auributıcrung, die zeıgt, da $\mathrm{B}$ man das Phänomen der Devianz mit relauv einfachen organuschen

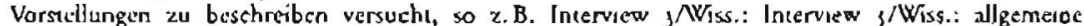
Unkennusis, aber ndas Volk silbst wciB Beschesd $x_{2}$ - Ganz interessante Antwonen crbrachee auch die Konírontation mit dem Allagsmychos, =der Russe ist im Grande seuncr Sccle cin Anarchist.. Dic Aлiworien bewegien sich erwariungsgemäß zwischen Zusummung und Ablehnung, aber of mit aufschluBreschen Begründungen: Anarchosmus bedeure Individualismus: dieser sei dem Russen lremo (Intervicw 4 /Wiss.); Anarchismus bedeute Kühnheit; dics sel dem Russen fremd usw. - Negativ demgegenüber lntcrnew 2 NWiss.: In sener negatıven Welesiche sei der Russe Anarchist.

62 S. erwa dic Studie von Eisenstade zur Auswahl tradiuonaler Symbolk in sozialistuschen Gesellschafien unter dem Gesichtspunkt ihrer sozialen Kampatibilitäl, S. N, Esensiadi, Sozsalismus und Tradition, in: ders./Y. Azman. Hrsg., Sozıalismus und Tradition, 1977, bes. ${ }_{4}$ f.; s. . übr. A. Cohen, Political Symbolism, Am. Rev. Anth. 8 (1979), 87f.; 5. jetzı auch U. Saranelli, Symbolische Politik, 1987.

63 C. Lane, The Rites of Rulers. Ritual in Indusunal Sociery: The Sovier Case, 198 ; M. Urban/). McClure, The FolkJore of State Socialism, in: Sovice Seudics XXXV $\left(198_{3}\right), 471$.

64 Tichomirov (Anm. 7/26.3.1987): Schutz gegen Bürokratısmus wic Kritiker; Aleksevo. Reche (Anm. \$).

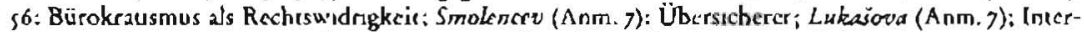
view 9/Prokuratur: Telefonrecht: Interview 12/Wiss: Warcncharakeer persönlichen Eigentums.

6) Najdenoe (Ann. 7/26.11.1986); Savickij (Anm, 7/4,12.1985): Alckscev, Reche (Anm. 8), 12; Andrecv (Anm. 7): Dzcržinskij's schmulzige Hände; Intcrvicw I6/W/Iss.: Markesozialismus als Befresung von den utopischen Elemencen des Sozıalismus. 
erlaubt, was vicht verboten ist ${ }^{b 6}$; Abschied wird genommen vom Prinzip "Plan um jeden Preis«; das Recht wird vom Instrument der Leitung der Gesellschaft zum eigenen Wert; und nicht mehr gure Gesetze, sondern die gute Ausführung von Gesetzen wird zum zentralen Ziel des Rechrssystems." ${ }^{61}$ Aus dem Vorzeigen des "deklarativen "Gesetzes wird das Prinzip des "Vorrangs des Gesetzes«; die Verfassung, lange totgeschwiegen bzw. wegen offenkundiger Bedeutungslosigkeit vergessen, taucht plötzlich im Gespräch und im juristischen Argument als feste Größe auf; Einstimmigkeit ist nicht mehr das einzig gültige Symbol der Einheit, und schließlich, die neueste und spektakulärste Symboländerung: Aus der wsozialiscischen Geseczlichkeit « wird die Konzeption des sozialistischen Rechtsstaats ${ }^{68}$ - ein jahrzehncelanges staatliches Sprachcabu ist damit gebrochen (und mirclerweile in aller Munde). Halten wir vor dem dritten Schritt zur Besinnung inne: Diese kritischen Worte sind authentischer sowjectischer Originalton - fast unglaublich! Zusammen mit den Reformen und Reformvorhaben, an deren Durchführung ich keinen Zweifel habe, ergibt sich das Bild einer immens dynamischen gesellschaftlichen Entwicklung.

\section{Alltag von gestern - die alten Selbstverständlichkeiten}

\section{Die Selbstwahmehmung der Juristen}

Unseren ersten Blick bei der Suche nach der Beharrlichkeir des Gestern werfen wir, wieder durch die Augen unserer sowjetischen Gesprächspartner, in einen imaginären Spiegel: Wie sehen sich die sowjetischen Juristen selbst? Schon bei der Betrachzung der Instizutionen wurden ganz handfeste Interessen an sozialem Aufstieg und Statusgewinn sichrbar; entsprechend unzufrieden ist man mit der augenblicklichen Situation in der Praxis (und auch in der Recheswissenschaft, deren bisheriges Wirken als zum größten Teil leere Scholastik empfunden wird) ${ }^{69}$ Zusätzlich sind auch jene unzufrieden, auf die sich die Kritik an den Institutionen konzentriert, vor allem also Prokurazur und Ermiztlungsbehörden. Man fühls sich ungerecbr behandelt, war man doch derjenige, der in der dunklen Zeit der Erscarrung die Fahne der Gesetzlichkeit troez unmöglicher Arbeirsbedingungen hochgehalten har. ${ }^{\circ 0}$ Interessanter als dieses Reagieren auf eine neue, ungewohnte Situarion ist jedoch die situationsunabhängige Selbstwahrnehmung. Diese versuchte ich in den Interviews anhand von drei Fragestellungen herauszudestillieren. Die erste betraf die Wahrnehmung des Verhältnisses von Jurist und Gesetz. Spätestens seit J. Esser, aber eigentlich schon viel länger wissen wir, daß der Richter/Jurist niche erwa der Mund des Gesetzes, sondern daß das Gesetz das Werkstück des entscheidenden Juristen ist, aus dem er vieles, wenn auch nicht alles formen kann.7' Das war natürlich auch

66 S. etwa-Lazarev (Anm. 7); Interview 2/Wiss.; Interview 13/Verwaltung: „Wir crlauben allcsk (was freilich bezweifeic werden darf)

6) Kondratäik/Kallistos (Anm. 7): kein Plan um jeden Press; Demokraue und Gesctz (Anm. 8): von guten Geseizen zu guten Geserzesausführungen; s. auch Tichamirov (A $\mathrm{mm} .7 / 26.3$ 1987): Ubergang von geseulichen zu vertraglichen Beztehungen; Solov'eva (Anm. 8): Rechu als eigener Wern.

68 Savickij (Anm.7/9.4.1987); Kudravcev (Anm. 7/27.8. 1987); Solov'eva (Anm. 8); Demokrnue und Gesecz (Arm. 8): Alekseev, Reclu (Anm. 8), 24, 77, 127, 139f,; Lann (Anm. 7/1 5.7.1987); Incerview 2/ Wiss.

69 S. dic Nachweise in den Anm. 18 bis so sowle etwa Alckseev, Reche (Anm.8), 15. 160, 163, zur Rechiswissenschait der Zukunfi; s. auch Rekunkov (Anm. 8) sowie Demokratic und Reche (Anm. 8); s. auch Piskarev (Anm. 7).

7o S. etwa Kudravicew (Anm. 7/27.8. 1987); Kar/Naxarenko/Mulikow (Anm. 7); Intcrvew 9/Prokuratur: - In der Zell der großen Gauncra.

7) J. Esser. Vorverständnis und Mcthodenwahl, 1972. 
schon so, bevor wir uns das eingestanden haben. Das Eingeständnis zeigt jedoch einen Gewinn an Subjektqualität der Juristen. In der UdSSR, so die Beancwortung der Frage nach dem Verhältnis Jurist/Gesetz, ist das Geserz immer noch vorgegebene, alles enthaltende, der Veränderung und Encwicklung durch den Richter nichı unterliegende Größe. ${ }^{72}$

Die zweite Fragestellung betraf ein oft übcrsehenes, weil ungern gesehenes (zu Recht) Element Menschlichkeit im Rechtssystem, nämlich den Rechtsanwendungsfehler. Die Justizkritik in der Tagespresse der UdSSR kennc keine Rechtsanwendungsfehler, die nicht entweder Willkür oder aber Formalismus wären. Die Attribucierung mache klar, daß hier ein systemwidriger, bösartiger Auswuchs in einem eigendich fehlerlosen System vorliegt, den man nicht sachlich behebt, sondern mit heißem Herzen bekämpfe und gnadenlos vernichter. Ich fragte also meine sowjetischen Gesprächspartner, ob eigenrlich zwischen den Excremen Willkür und Formalismus auch ein normaler Rechesanwendungsfehler denkbar sei. Verblüffenderweise war die Frage überhaupt nur zwei von etwa 30 Interviewpannern auf Anhieb verständlich; auf Erklärungen hin leuchcete zwar auch den übrigen dieser nicht maximalistische, einfache Rechtsfehler ein; die meisten beeilten sich dann aber, mir klarzumachen, daß solche Fehler die absolute und verschwindende Ausnahme seien - was selbst bei Berücksichrigung anderer Fehierverteilung wegen der gesehenen Besonderheiten des sowjetischen Rechtssystems kaum haltbar sein dürfte. ${ }^{73}$

Die dritte Frage schließlich lautete schlicht: Was ist ein guter Jurist. Auch hier waren die Antworten (zumindest für mich) erstaunlich. Zunächst einmal differenzierten überhaupt nur zwei Gesprächsparner nach juristischen Berufen. Angeboren wurde mir zum einen eine breice Palecte buncer, aber immer nur sehr vereinzelt aútauchender Eigenschaften: offen für Kririk, erfahren, ein guter Taktiker, gesetzesgchorsam, mic Lebenserfahrung und Inruirion, klar denkend, veranrwortlich und harcnäckig, sich selbsc und andere achtend, mit geistiger Reinheit, ein guter Kommunist, wenn auch niche unbedingt Parteimitglied (einmal), mild und barmherzig, mit hoher politischer Kultur und breiter Allgemeinbildung. All diese Antworten kamen höchstens dreimal. Andere Eigenschaften wurden von mindestens einem Dritzel der Gesprächspartner genannt, vor allem gure Rechtskenntnis, hohe Professionalität und Gerecheigkeirsliebe. Der gemeinsame Nenner jedoch, den fast alle Gesprächsparner in dieser oder jener Weise ansprachen, war die Moral: ein gurer Mensch, ein ehrlicher Mensch, ein Mensch von hoher Moral, das sei ein guter Jurist.

\section{Die Kontinutät der Grundwertungen}

Unsere Ausgangsvermutung gehe davon aus, daß sich Änderungen im Bereich der Einstellungen, Änderungen unter der Oberfläche langsamer einstellen als jene im

72 Die sowjetıschen Gesprächsparıner zeigeen hier selır herkömmliche Vorstellungen in ihrer Selbstwahr-

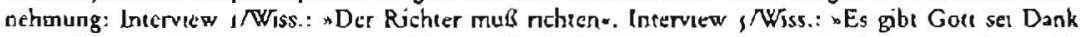
einen Obersten Sowjeta. Intervicw 6/Richter: Rechesforbildung, sowcit und rolange es micht an dic gehejligıen Grundlagen gche/möglichst genauc Umschrcibung durch den Gesetzgebtr. Interview of Prokuratur: "Vor dem Gesetz den Kopl beugen . Iniervicw 1:/Prokurarur: "Das Geselz zwingt und ubsraeugra, - Dementsprechend ein sonst manchmal durchschimmerndes Selbsiverständnis der Machtlosigkest, ctwa in der Aursage otheoretisch selbstverständlich, abcr prakusch ... , so ctara lnuerview 14 ' Wiss.; Intcrvicw $16 /$ Wiss.

73 Sehr häufig kam die Annworı, daß (unter Bezug auf en Lenin-Zilat) Wrilkür eun Versioß gegen den Buchstaben und den Geist des Gesetices sel, Formalismus dagegen nur ein Verstoß gegen den Geist des Gesetzes: so z. B. Inecrview a/Wiss.; Inecrnew s NWiss.; Intervew i $/$ Wiss. Selsr háufip land sich auch die Glerchserzung Willkür/Bürokrausmus, so z. B. Intervew 6/Richecr, der im wetteren auch selbst Fehlurteile als willkürlich bezeichnere; Interview 8/Richter: Rechisichler, wenn memand darunter gelinten habe (?). Die Zielncheung auf den normalen Ruchesanwendungsfchler crkannte man sofort, in lnerview $>$ /Advokatue. 
strukturellen oder sonst sichtbaren Bereich. So kam es denn auch in allen Interviews kontinuierlich zum Wiederhochspülen der alten Selbstverständlichkeiten, der Fraglosigkeit gewisser - alccr - Vorstellungen von gut und schlechr. Besonders sichtbar wurden diese unteren Lagen unseres Palimpsests beim Gespräch über das „Gesetz über die individuelle Arbeitstärigkeit . Grundsätzlich wurde das Geserz von allen gurgeheißen, aber: Eigentlich sei solche Arbeit, dic nicht für den Staat, nich für dic Gesellschaft gelcistet werdc, negativ, unmoralisch, ein illegitimes Kind des Sozialismus. Es gehe nicht an, daß Gesellschaftsmirglieder Verdienste in unmoralischer Höhe hätten; zu hoher Verdienst seien sog. Nichtarbeitseinkünfte (und damic strafbar. $)^{7 /}$ Ein Problem sei es auch, wenn mit solcher individucller Arbeicstärigkeit eine Tärigkeit ohne Nuczen für den Menschen ausgeübt werde: Deswegen müsse die Verwaltung den gesamten Bereich der individuelien Arbeitstätigkeit überwachen und ausforschen und so die Gefahren abwehren, die die Tàtigkeit als solche für das Wohl von Gesellschaft und Staat zwingend beinhalte. ${ }^{\text {7s }} \mathrm{Z}$ war sei alles erlaubr, was niche verboten sei, aber cben doch nur in den Grenzen des moralisch Erlaubten wenn nicht überhaupt das Verhältnis von Erlaubnis und Vcrbor bei der individucllen Arbeitstärigkeit zum repressiven Verbor mit Befreiungsvorbehalt umgedcutct wurde. ${ }^{76}$ Individuellc Arbeitstätigkeit sei eben gerade erwas anderes als Tätigkeit im Kooperativ: Diese sei gesellschaftliche (und damit wohl automatisch nützliche) Arbeitstärigkeit. ${ }^{77}$

Ähnliche Grundwertungen fanden sich auch im Bercich der staarlichen Volkswirtschaft: Es gebe keine Gesetzesverletzung von Wirtschaftsnormen, die nicht egoistische Motive habe: Ökonomische Handlungsformen seien unmoralisch, cine Unterwanderung wirtschaftlicher Moral. So könne es auch kein Pardon für Gesetzesverletzungen geben, auch wenn sie volkswirtschaftlichen Nutzen brächten. ${ }^{78}$ Zitat: Es gibt auch ein moralisches Problem neben dem wirschaftlichen; und: $80 \%$ Verdienst sind schon Kapitalismus. ${ }^{79}$ Durchzogen von einer tiefen Mißbrauchsphobie und einem grundlegenden Mißraucn, einer allgemeinen Schädlichkeitsvermutung sind auch sonstige Aussagen, etwa zum ${ }$ Gesetz über die gerichtliche Bcschwerde gegen unrechtmäßige Handlungen von Amtspersonen«. Dem Geist des Gesetzes nach könne zwar alles beklagt wcrden: Da müsse es aber doch Grenzen geben. Der Rechrsmechanismus dürfe nicht zu persönlichen Zwecken ausgenützt werden; bestimmte Handlungen müßten selbstverständlich unterbunden werden, unberechrigte Kritik sei zu verfolgen. Demokratie sei nicht die Möglichkeit des Egoismus! ${ }^{180}$

74 Interview is/Richrer: Nichtarbeitscinkünfli; Intervicow r6/Wiss.; lllegumes Kind; Intervicov 8/Richeer: Mißbraucl durch geschickte Menschen; Intervicw 17/Wiss., Inecrvicu 14/W/iss.: uirsoz.1aliseiseh und uninoralisch als Beıvernung durch die älerc Generalion; (ntervicw 18 Niss.: Es störe, da 3 dic Menschen nicht mehr für den Staat arbelteten, cs musse eune Grenze für hohe Verdicnsic geben: Aleksel"w, Reche (Anm. 8): Beconung der aligemeunen Pflichrenbindung des Buirgers.

75 Bareckij (Anm. 7); Kanpec (Anm. 7): Nichestun als unmoralisch. Nichistuer als A3koholiker.

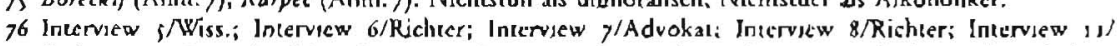
Prokuratur: Gesunder Menschenversiand; Interview 16/Wiss.: Eine reune Erlaubnis sei noch kem (subjeknves) Reche; lnecn'iew $17 /$ Wiss.: Laut empinscher Untersuchungen hieleen gerade dic Zielgrupper (Renener) das Gesece lür moralisch venverflich. - Haufig auch die Poseulicrung ennes Ermessens/

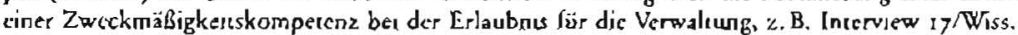

77 Intervicw 1s/Richter; Interview 16 Noiss.

78 Interview Ir/Prokucatur; Novikov (Anm. 7); Najdenov (Anm. 7/1.8. 1986); Lukajova (Anrn. 7 ).

79 Internitw ig/Wiss.: $80 \%$; Intervicw 18/Wiss.: Moralisches Problem; dies wurde freilich auch knisicre, so etwa Intervica 12/Wiss.: Úbermäßige Gewinne müßten über Stcuen abgeschöpfi werdes.

so Demokratse und Gesetz (Anm.8); Interview 18/Wiss.: Rechelich nehe faßbarc Rechesumgehung:

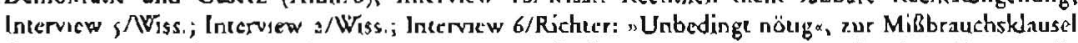
des Ar, yo Verwalcungsgerichesgesclz: Inerview g/Prokumiur, mil dem verstärkenden Hinweis, die affentiche Mcinung sel dagegen: hier auch das Dikrum, Jas Volk kenne zwar seune Rechre, nicht aber seine Pflicheen; cbenso Intervicw is/Richter; Interview it/Wiss.: r Rechesverletzung, dic dem gesunden Mensehenversund/dem Geist des Gesezzes widerspreche, als sndirekte Gesetzesverletzunga. 
Der Theorie der Unschuldsvermutung, um weitere Beispiele zu nennen, steht die Selbstverständlichkeit der Annahme der Strafbarkeit noch nicht Verurteilter gegenüber; Zehntausende von Verbrechern sieht man bei Aufweichung des Straíprozeßrechts, bei strenger Rechusbindung der Ermitrlungsbehörden, in Freiheit. ${ }^{81}$ Die Verfassung hat zwar sclbscvcrständlich unmittelbare Geltung; man würde sie aber sie in der praktischen Arbeic anwenden (Zitat eincs Richters). ${ }^{{ }^{3}}$ Die Gerechtigkeit einer Entscheidung verwandelt sich schließlich unversehens in ihre Zweckmäßigkeit: Vorstellungen von Gesundheit, von gesundem Rechtssystem gegenüber den kranken Abweichungen, den psychisch kranken Bürgern, die sich abweichend verhalten, demonstrieren wie auch die Bezüge zur Moral die Existenz einer das Recht relativierenden Metaebene ${ }^{\delta_{3}}$; Zitat eines Richters am Beispiel der Aufhebung einer rechrswidrigen Verwaltungsentscheidung: Das ist eine moralisch schwierige Entscheidung ${ }^{8}{ }^{8}$

\section{Die Modalitäten des Rechussystems}

$\mathrm{Zu}$ diesem doppelbödigen Orientierungssystem der Juristen kommen spezifische, für uns ungewöhnliche Modi der Gesetzesimplementation und juristischen Arbeit. Gesetze werden in der UdSSR (in der Theorie; die Praxis ist sowieso anders) nicht einfach befolgt: Strenge, strengste Erfüllung der Gesetze ist angesagt, nicht auch die allerkleinste Abweichung von Gesetzen soll geduldet werden; entscehen muß ein Geist der Unduldsamkeit gegenüber Gesetzesverletzungen; das Gesetz, das natürlich auf der Grundlage riefer und tiefster Erforschung seiner soziologischen Wirksamkeitsbedingungen und der gerichtlichen Praxis gemacht wird, muß unerschütrerlich sein. ${ }^{83}$ Jegliche, auch kleinste Unkiarheiten im Gesetzeswortlaut müssen ausgeschlossen sein. Beliebige Gesetzesverletzungen, auch minimalste, sind gnadenlos zu verfolgen; denn auch in der kleinscen Verletzung der Gesetze lauer die Gefahr des Zusammenbruchs der gesamten Rechtsordnung ${ }^{86}$, die Gesetze sind *heilig* (Zitat).

8. Piskarev (Anm. 7); Kondraicik/Kallistov (Anm. 7); Andreev (Anm. 7); s. auch cbd., Der Advokat als Fcind; Savickij (Anm. 7/4, 12, 198s).

82 Intervicw 6/Richter; noch weilergehend Intervicw 7/Advokat: Veriassung als Theore: dem enespncht sine Unwilligkell vieler anderer Gesprïchspanner, sich auf veriassungsechtliche Argumeneacuanen

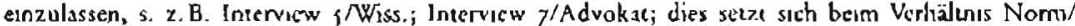
Verordnung for, so crwa Intervic' 18 /Wiss.: Selbstverständlich konnc cinc Verordnung cın Gesetz einengen und modifizaeren.

83 Intcrview s $N$ Wss.: Zweckmäßigkeir und Gerechugbeıt seien für den Bürger eins, was dann kurz darau\} als Bewers rechtlichen Nihilismus gewertet wurde; Intervicw 8/Richter: "Nervlich angespannte Bürger a; Intervicw 13/Verwaltung: Psychisch kranke Burger (anl. der Beschwerden); Interview 19/Richter: Psychische Defekie bei denen, dic behaupten, der Rurse sei Anarchist (s, zu diescr Fragestellung Anm.61); Intervicw is/Wiss.: Sozialc Gesundhett - Übertragung organischer Vorstellungen aul die Gesellschafi; s. auch Rekunkov (Anm. S): Idenurät von Cisserdichkeit und Parteilichkerk.

8. Inetrvew 6/Kicheer; diese Vermengung von moralischen und rechelichen Kategorien fand sich auch sonst in den Interviews, so ecwa Ineerview $2 / W_{i s s}$ S. I. ube. Perrudin $(\Lambda n m .7)$; Lukasova $(\Lambda \mathrm{nm} .7)$. Ähnlich unsaubere Trennung von Kategorien finden sich auch be der Idenuilizserung von Rechisscaat bzw. rechesseaselicher Ennwicklung und Demokracic; tine der Standandfragen in den Inservews betraf diese Vermischung, und kaum eincr der Gesprächspartner zeıgec hıcr (zumindesı aul erstes Befragen) Distanzicrungen ingendwelcher Art: eine Ausnahme etwa in Intervicw ; Niss.

8f Interview 9/Prokuratur; Interview Io/Wiss.; Inerview 11/Prokuratur; Sucharev (Anm. 7): Ehrfürchtige Bezichung xum Gericht; cbenso Anaskem (Anm.7/25.9.1985): Boreckij (Anm. 7): Durchüuhrung der Relormen mil eiscrner Hand; Kudraveer (Anm. 7/4. 10. 1985): Tichomirov (Anm. 7/26. 3. 1987), Baranov (Anm.7); Rekunkos (Anm. 7), ro: Allgimcine Verachiung gegenüber Rechisbruch; Filacos (Anm. 7): Lukasoove (Anm. 7); Ole.jnik (Anm. J/30.5. 1986).

86 Interview 8/Richter: Serenge Strafen fur Willkuir und Formalismus bet Rechtsanrucndung: Nagdenov (Anm. 7/29.3.1983); Alekseev. Reche (Anm.8), I21., 73 u.0.; Rekunkor (Anm. 7), 13: allgemcine Vcrachuring gegenuber Rechesbruch.

8) Alekseev, Recht (Anm. 8), 132: Tichomirov (Anm.7/3.1.1986); interessant auch Interview i6/Wiss.: 
Auch die neuen Gesetze in der Konsequenz der Reformpolitik werden nicht ganz pragmatisch qua Entscheidung erlassen: Die Zeit ist reif, die Notwendigkeit ist herangereif, die Geschichce gebietet geradezu, dieses oder jenes $7 u$ tun. ${ }^{88}$ Und was schließlich den Bürger und sein wie gesehen schleches Verhältnis zum Gesecz und Rechussystem betriffe: Er muß crzogen werden, er muß die juriscische Reife erwerben, das Rechessystem muß propagien werden ${ }^{89}$ (mit anderen Worten: es kann nicht für sich selbst werben). Signifikant waren schließlich auch die Tabus, die innere Zensur bei den Gesprächspannern und sonstigen Interviews: „Wir reden jetzt of davon « (Zitat), so eine häufige Floskel, mit der bestimmte Mißstầnde im Rechtssystem angesprochen wurden, und nicht etwa: dieses oder jenes ist entdeckt worden: Die Sprache ist of verrärerisch genau. Bemerkenswert auch die Regionalisierung der Mißstände: Das Phänomen der Bestechung, der Strafneigung, der Einflußnahme der Exekurive auf die Gerichee ist etwas, was immer irgendwo da draußen, an einem skonkreten Ortı, in der Kom; ASSR etwa geschieht - nicht aber überall -, und es geschieht an der Peripherie, d.h. eben nicht im - offensichtlich tadelsfreien - Zentrum..${ }^{\circ}$ Und schließlich: „Unser Land iss so groß - die den Einzelnen erdrückende Größe schlägt auf die Gesellschaft durch.9r

Die alten Grundwcrungen werden also durch eine spezifische Modalität ergänzt: Die Zielsetzungen sind nicht rcalistisch, sondern illusionär, ja absurd; der Zugang zu den Dingen ist nicht flexibel, sondern durch zu hoch gesetzte Ziele völlig starr; die Beziehung zum eigenen Gegenstand ist nicht sachlich, sondern aułgeregt. Der eben gesehene Doppelmaßstab findet seine Enssprechung im illusorischen Modus: Die Potemkin'sche Fassade des Rechtssystems sorgt dafür, daß der handelnde Jurist eigentlich immer außen davorsteht, entweder vorne bei der prachtvollen Illusion oder dahinter bei den ärmlichen Hütten des Alltags. Auch hierfür hat die russische Sprache natürlich ihr Wort - Zitat: unser bekannter Maximalismus.

\section{IV. $\operatorname{Schlu} \beta$}

Die gefundenen Fakten sind vieldeutig. Welche der viclen möglichen zusammenfassenden Würdigungen kann nun das Resümee sejn. Die Gedanken sind, wie wis alle wissen, frei: Nichr etwa die Meinungsireiheit ist schuczbedürftig, wohl aber die Freiheic der Äußerung, der Betätigung, der Verwirklichung von Meinungen. Zur Freiheit des Menschen gehör im Falle ciner repressiven Umwelt die Schaffung eigener, verborgener Gegenwelten: Auch diese Gegenwelten reproduzieren aber regelmäßig bis zu einem gewissen Grad die Strukturen und Inhalte jener Umweit, aus der man geflohen ist. Eine gesellschaftliche Reform (dies gilt auch für Reformen

Kritik an Veraalungsgenehtsgesclz, weil es keıne Verantwortichkent festlege: Es müsse doch eme Verantwonlichkest existieren - dies in cunce Gesellschaft, deten Grundserukrur auf Gememlast und eben nuche auf individuefle Verantwortlichkest ausgericheet isL Incerview 18 Wiss.: Schreckensvorseellung abwerchender ersunstanzlicher Genchesentscheidungen.

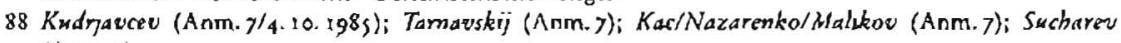
(Anm. 7).

89 Interview 8/Richter: Intervicw 23/Richer: „Von oben aus (Regienng/Panei) sei alles sichibark.

go interview 17 /Wiss.: Wir reden jeizt oft davan; Boreckij (Anm. 7); Intervicw 14 NWiss; Intervicw is' Richtcr; Interview I6/Wiss. - wobel diese Zentrum-Penphene-Differenzıcrung häufig mit einer qualiuxiven Abwcroung der Penpherie zusammenfalle: besonders deutlich noch Ynterview $18 /$ Wiss.; die innere Zensur wurde ausdrücklich als solche bezerchnce, etwa Intervicw ${ }_{12}$ NWiss.

91 Interview is Richer: Es versiche sich, daß auch alte Symbole unter der Hand aufrauchen, s. ctwa Alekseev, Rechr (Anm. 8), 26, 65 ч. ö.; Solov'eva (Anm. 8): Xeine Gewaleenteilung: Gribrnov (Anm. 7): Ordnung ist Ordnung; es $\mathrm{kam}$ auch zu eigenartigen Kombinationen, erwa Interview 8/Richter: Patriousmus als Rechiskulur. 
durch walte Eliten wie im Falle Gorbačevs) ist zu einem großen Teil die Schaffung einer neuen Welt aus alter Wele und alter Gegenwelt. Auch die Reformen im Rechtssystem der UdSSR weisen diese nicht zu umgehende Konkordanz mit der eigenen Vergangenheit aut: Deren Besonderheic besteht nun darin, daß die alten Inhalte Elemente des Gelingens und Elemente des Scheiterns jenes Versuches einer neuen Gesellschaftsordnung widerspiegeln, der 1917 untemommen wurde. Starke Gemeinschartsbezogenheit, dezidierte Vorstellungen über eine gleichmäßige Verteilung gesellschaftlichen Reichtums, die Selbstverständlichkeit des Anvisierens von hohen, höchsten, von utopischen Zielen, die Sicht des Einzelnen als lebensweltlichganzheiclich und nicht nur als Kompendium von Rollen, das Mißtrauen gegenüber dem "Nichr-Richtigen " und dahinter liegende Grundannahmen einer Polarität richrig/falschr - all das ist der ganz reale Sozialismus. Auf der anderen Seite stehen die Elemente des Scheiterns; sie sind die selbstverständlichen Überlebensbedingungen in einer repressiven gesellschaftlichen Umwelt. In einer solchen Umwelt brauchr der Einzelne viele Leben, und zwar nebeneinander, um einigermaßen leben, manchmal auch überleben zu können: Repressive gesellschaftiche Umwelten führen zum Lebensmodus einer kleinen Schizophrenie. Dies fanden wir auch in der UdSSR: Ich erinnere an die Selbstverständlichkeit der Existenz über- und unterlagernder Verhaltensmaßstäbe (Recht/Moral, Gesetz/Instruktion), an die Automatik des simultanen Einbeziehens und Ausgrenzens (Zentrum/Peripherie), an die fraglose Aufspaltung der Welt in jene, die Subjekt und jene, die Objekt sind. Gegenüber dem realen Sozialismus und den mit ihm verbundenen Wertungen beachte ich das Prinzip respektvoller Nichreinmischung. Zu dem ,Überfluß uncerschiedlicher Welrenc möchte ich jedoch zum Abschluß noch eine augenzwinkernde Keczerei äußern: Wenn man in der UdSSR eingesehen hat, daß Entscheidungen über Rechtmäßigkeit keine moralischen Entscheidungen sind und daß ein guter Jurist nicht unbedingt ein guter Mensch sein muß, mit anderen Worten: $\mathrm{da} ß$ weniger mehr ist und daß eine Welt ausreicht, dann hat man auch das verändert, was niemand auf einmal ändern kann. 